\title{
Brane-world solutions, standard cosmology, and dark radiation
}

\author{
Shinji Mukohyama \\ Department of Physics and Astronomy, University of Victoria \\ Victoria, BC, Canada V8W $3 P 6$
}

(August 21, 2018)

\begin{abstract}
New exact solutions of brane-world cosmology are given. These solutions include an arbitrary constant $C$, which is determined by the geometry outside the brane and which affects the cosmological evolution in the brane-world. If $C$ is zero, then the standard cosmology governs the brane-world as a low-energy effective cosmological theory. However, if $C$ is not zero, then even in low-energy the brane-world cosmology gives predictions different from the standard one. The difference can be understood as "dark radiation", which is not real radiation but alters cosmological evolutions.
\end{abstract}

PACS numbers: 04.50.+h; 98.80.Cq; 12.10.-g; 11.25.Mj

Superstring theories [1] have been considered as strong candidates for the theory of everything. Among them, the $E_{8} \times E_{8}$ heterotic superstring theory has been traditionally considered as the most relevant one for phenomenology since $E_{8}$ includes the gauge group of the standard model as a subgroup. On the other hand, recent progress on dualities between various string theories and M-theory made it possible to investigate strong coupling behavior of them [3]. In particular, Hořava and Witten [4] showed that strong coupling limit of the $E_{8} \times E_{8}$ superstring theory can be described by the 11-dimensional supergravity, which is considered as a low-energy effective theory of M-theory, compactified on $S^{1} / Z_{2}$ of large radius. In their scenario, the coupling constant of the heterotic superstring theory is interpreted as radius of $S^{1} / Z_{2}$, and matter fields are confined on two 10-dimensional timelike hypersurfaces which correspond to fixed points of $S^{1} / Z_{2}$. Moreover, after compactification on a Calabi-Yau manifold, the theory of matter on each of the two fixed-point hypersurfaces, which is 4-dimensional after compactification, is $N=1$ supersymmetric $E_{8}$ gauge theory. There is evidence that over a wide range of energy scale this 5-dimensional picture holds [5.6]. Thus, in this picture our 4-dimensional universe is one of the 4-dimensional timelike hypersurfaces, or the world volume of a 3-brane, in 5-dimensional spacetime compactified on $S^{1} / Z_{2}$. All matter fields including those of the standard model is confined on the 3 -brane.

Although this picture seems satisfactory as a derivation of the theory of matter fields in 4-dimension, it is expected at least for a moment that Kaluza-Klein modes of gravitational field might spoil it. The large radius of $S^{1} / Z_{2}$ implies that mass gap of Kaluza-Klein modes is small and that there appears a tower of infinite number of light Kaluza-Klein particles, which contradicts experiments.

Recently, it was found that the above embarrassing expectation is not correct. Randall and Sundrum [7 showed that, for small perturbations around a flat brane-geometry, zero modes of gravitational field, which are massless on the brane and which correspond to gravitational field on the brane-world, are trapped on the brane due to the brane tension. Moreover, no Kaluza-Klein modes which are massive in the 4-dimensional theory in the brane-world are strongly coupled to the gravitational zero modes and matter fields on the brane. Therefore, Hořava-Witten scenario seems satisfactory as a derivation of the theory of weak gravitational field as well as matter fields in 4-dimension.

Now, one of the problems in string theories and M-theory is that there is no direct experimental or observational evidence of them. In this respect, it seems very effective to investigate cosmological implications of these theories, since the universe is considered to be in very high energy at its early stage. Since the Hořava-Witten scenario seems very satisfactory as explained above, many authors investigated cosmology in the 3-brane world based on the scenario [8,9]. (Many complementary works have been done on black holes [10, 11], non-linear analysis [12], gravitational force between two bodies [13], and AdS/CFT correspondence [14.) However, no global solution in 5-dimension which gives the standard cosmology in the brane world have been given yet. The purpose of this paper is to give it and investigate its cosmological implications.

As a five-dimensional geometry, let us consider a metric of this form

$$
d s_{5}^{2}=-N(\tau, w) d \tau^{2}+R(\tau, w)\left(d x^{2}+d y^{2}+d z^{2}\right)+d w^{2} .
$$

We consider a negative cosmological constant, which is interpreted as an expectation value of 3-form field in HořavaWitten scenario, and seek a general solution of the Einstein equation $G_{b}^{a}=3 k^{2} \delta_{b}^{a} / 2$ in bulk for this ansatz. Here, $k$ is a positive constant. It is easy to calculate the Einstein tensor for this metric, and the result is

$$
G_{\tau}^{\tau}=\frac{3}{4 N R^{2}}\left(2 N R R^{\prime \prime}-\dot{R}^{2}\right)
$$




$$
\begin{aligned}
G_{w}^{\tau} & =-\frac{G_{\tau}^{w}}{N}=\frac{3}{4 N^{2} R^{2}}\left(2 N R \dot{R}^{\prime}-N \dot{R} R^{\prime}-N^{\prime} R \dot{R}\right), \\
G_{w}^{w} & =\frac{3}{4 N^{2} R^{2}}\left(N^{2}{R^{\prime}}^{2}-2 N R \ddot{R}+\dot{N} R \dot{R}+N N^{\prime} R R^{\prime}\right), \\
G_{j}^{i} & =\frac{\delta_{j}^{i}}{4 N^{2} R^{2}}\left(2 \dot{N} R \dot{R}-4 N R \ddot{R}+2 N N^{\prime} R R^{\prime}-N^{2} R^{\prime 2}+N \dot{R}^{2}+4 N^{2} R R^{\prime \prime}+2 N N^{\prime \prime} R^{2}-N^{\prime 2} R^{2}\right),
\end{aligned}
$$

where dots and primes denote derivatives with respect to $\tau$ and $w$, and $i$ and $j$ denote $x$ or $y$ or $z$. Since the $(\tau w)$-component of the Einstein equation, $G_{w}^{\tau}=0$, becomes $\left(\dot{R}^{2} / N R\right)^{\prime}=0, N(\tau, w)$ is written as

$$
N(\tau, w)=f(\tau) \frac{\dot{R}^{2}(\tau, w)}{R(\tau, w)},
$$

where $f(\tau)$ is a positive function of $\tau$. Substituting Eq. (6) into Eq. (2), the $(\tau \tau)$-component of the Einstein equation, $G_{\tau}^{\tau}=3 k^{2} / 2$, becomes $R^{\prime \prime}-k^{2} R=1 / 2 f$. Hence, $R(\tau, w)$ is written as

$$
R(\tau, w)=\alpha(\tau) \cosh (k w)+\beta(\tau) \sinh (k w)-\frac{1}{2 k^{2} f(\tau)},
$$

where $\alpha(\tau)$ and $\beta(\tau)$ are functions of $\tau$. For this form of $R(\tau, w)$ it is easy to confirm by using Eq. (5) that the (ij)-components of the Einstein equation, $G_{j}^{i}=3 k^{2} \delta_{j}^{i} / 2$, are satisfied. It is easy to show that the remaining equation $G_{w}^{w}=3 k^{2} / 2$ becomes $\partial_{\tau}\left(4 k^{4}\left(\alpha^{2}-\beta^{2}\right)-1 / f^{2}\right)=0$. Therefore, $f(t)$ is written as

$$
f(\tau)=\frac{1}{2 k^{2} \sqrt{\alpha^{2}(\tau)-\beta^{2}(\tau)+C}},
$$

where $C$ is a constant.

Note that two functions $\alpha(\tau)$ and $\beta(\tau)$ and the constant $C$ are arbitrary. However, these degrees of freedom should include degrees of freedom of coordinate transformations. In fact, we can define a new time variable $t$ and a function $a(t)$ of $t$ so that the metric obtained above is transformed to the following form.

$$
d s_{5}^{2}=-\frac{\psi^{2}(t, w)}{\varphi(t, w)} d t^{2}+\varphi(t, w) a^{2}(t)\left(d x^{2}+d y^{2}+d z^{2}\right)+d w^{2},
$$

where $\psi$ and $\varphi$ are given by

$$
\begin{aligned}
& \psi=\cosh (k w)+2 k^{-2}\left(H^{2}+\partial_{t} H\right)(\cosh (k w)-1) \pm \frac{1+2 k^{-2}\left(2 H^{2}+\partial_{t} H\right)}{\sqrt{1+4 k^{-2} H^{2}+C a^{-4}}} \sinh (k w), \\
& \varphi=\cosh (k w)+2 k^{-2} H^{2}(\cosh (k w)-1) \pm \sqrt{1+4 k^{-2} H^{2}+C a^{-4}} \sinh (k w)
\end{aligned}
$$

(two signs of terms including $\sinh (k w)$ should be taken to be the same) and $H(t)$ is determined by $a(t)$ as

$$
H=\frac{\partial_{t} a}{a} .
$$

The new time variable $t$ and the function $a(t)$, which is arbitrary for the moment, are defined by

$$
\begin{aligned}
\frac{d t}{d \tau} & =\sqrt{N(\tau, 0)}, \\
a(t) & =\sqrt{R(\tau, 0)} .
\end{aligned}
$$

Therefore, the solutions of the Einstein equation $G_{b}^{a}=3 k^{2} \delta_{b}^{a} / 2$ which have been obtained by using the ansatz (11) are written in the form (9) with Eqs. (10-11). The function $a(t)$ is arbitrary for the moment, but the evolution equation of it will be determined below by so called Israel's junction condition 15 at the brane. On the other hand, the constant $C$ will be arbitrary to the end.

Let us suppose that a 3-brane is at $w=0$. Hence, because of the $Z_{2}$-symmetry, the geometry in bulk should be invariant under parity transformation $w \rightarrow-w$. Thus, the 5 -dimensional metric is given by Eq. (9) with

$$
\begin{aligned}
& \psi=\cosh (k w)+2 k^{-2}\left(H^{2}+\partial_{t} H\right)(\cosh (k w)-1) \pm \frac{1+2 k^{-2}\left(2 H^{2}+\partial_{t} H\right)}{\sqrt{1+4 k^{-2} H^{2}+C a^{-4}}} \sinh |k w|, \\
& \varphi=\cosh (k w)+2 k^{-2} H^{2}(\cosh (k w)-1) \pm \sqrt{1+4 k^{-2} H^{2}+C a^{-4}} \sinh |k w|
\end{aligned}
$$


(As in Eq. (10), two signs of terms including sinh $|k w|$ should be taken to be the same.) Note that the induced metric on the brane at $w=0$ is

$$
q_{\mu \nu} d x^{\mu} d x^{\nu}=-d t^{2}+a^{2}(t)\left(d x^{2}+d y^{2}+d z^{2}\right)
$$

and, thus, is the flat FRW metric with the scale factor $a(t)$.

Now, in order to give evolution equations of $a(t)$, we use so called Israel's junction condition [15]:

$$
\begin{aligned}
{\left[q_{\mu \nu}\right] } & =0, \\
{\left[K_{\mu \nu}-K q_{\mu \nu}\right] } & =-\kappa_{5}^{2} S_{\mu \nu},
\end{aligned}
$$

where $[X]$ denotes $\lim _{w \rightarrow+0} X-\lim _{w \rightarrow-0} X, S_{\mu \nu}$ is the surface energy-momentum tensor on the brane, and the extrinsic curvature $K_{\mu \nu}$ of the constant- $w$ hypersurface and its trace $K$ are defined by $K_{\mu \nu} \equiv q_{\mu}^{\alpha} q_{\nu}^{\beta} \nabla_{\alpha}^{(5)} n_{\beta}$ and $K \equiv K_{\mu}^{\mu}$. Here, $\nabla_{\alpha}^{(5)}$ is the five-dimensional covariant derivative with respect to the metric we have obtained and $n^{\mu}$ is the unit normal to the constant- $w$ hypersurface given by $n^{\mu} \partial_{\mu}=\partial_{w}$. Since Eq. (15), which represents a gauge condition, is satisfied automatically for our metric, we shall concentrate on Eq. (16).

We assume that the surface energy-momentum tensor is a sum of a surface tension term and a perfect fluid term:

$$
S_{\mu \nu}=-\lambda q_{\mu \nu}+T_{\mu \nu}
$$

where

$$
T_{\nu}^{\mu}=\left(\begin{array}{cccc}
-\rho(t) & 0 & 0 & 0 \\
0 & p(t) & 0 & 0 \\
0 & 0 & p(t) & 0 \\
0 & 0 & 0 & p(t)
\end{array}\right)
$$

As in Refs. [7], the surface tension is assumed to be related to the bulk cosmological term as

$$
\lambda=\mp 3 \kappa_{5}^{-2} k \text {. }
$$

This relation arise in the five-dimensional effective theory of the Hořava-Witten scenario [4] 16 . It would be worth while mentioning that deviation from Eq. (19) will produce non-zero cosmological constant in the brane-world.

Since $K_{\nu}^{\mu}-K q_{\nu}^{\mu}$ is given by

$$
\begin{aligned}
K_{\tau}^{\tau}-K & =-\frac{3}{2} \partial_{w} \ln \varphi, \\
K_{j}^{i}-K \delta_{j}^{i} & =-\frac{1}{2}\left(2 \partial_{w} \ln \psi+\partial_{w} \ln \varphi\right) \delta_{j}^{i},
\end{aligned}
$$

the left hand side of Eq. (16) is calculated as

$$
\begin{aligned}
{\left[K_{\tau}^{\tau}-K\right] } & =\mp 3 k \sqrt{1+4 k^{-2} H^{2}+C a^{-4}}, \\
{\left[K_{j}^{i}-K \delta_{j}^{i}\right] } & =\mp k \frac{3+4 k^{-2}\left(3 H^{2}+\partial_{t} H\right)+C a^{-4}}{\sqrt{1+4 k^{-2} H^{2}+C a^{-4}}} .
\end{aligned}
$$

Thus, Eq. (16) becomes

$$
\begin{aligned}
3 k\left(\sqrt{1+4 k^{-2} H^{2}+C a^{-4}}-1\right) & =\mp \kappa_{5}^{2} \rho, \\
3 k\left(\frac{3+4 k^{-2}\left(3 H^{2}+\partial_{t} H\right)+C a^{-4}}{3 \sqrt{1+4 k^{-2} H^{2}+C a^{-4}}}-1\right) & = \pm \kappa_{5}^{2} p .
\end{aligned}
$$

These are the cosmological equations on the brane-world. These equations combined with an equation of state determines $a(t), \rho(t)$ and $p(t)$ uniquely as functions of time, provided that suitable initial conditions are given. The constant $C$ is still arbitrary and its physical meaning as "dark radiation" will be discussed below.

The undetermined signs in the above equations will be determined by considering a low-energy limit as follows. In order to seek low-energy effective cosmological equations, we should consider the case in which $k^{-2} H^{2} \ll 1$ and $\mathrm{Ca}^{-4} \ll 1$. In this case, Eqs. (22) become 


$$
\begin{aligned}
H^{2} & =\mp \frac{8 \pi G_{N}}{3} \rho-\frac{C k^{2}}{4 a^{4}}, \\
\frac{\partial_{t}^{2} a}{a} & = \pm \frac{4 \pi G_{N}}{3}(\rho+3 p)+\frac{C k^{2}}{4 a^{4}},
\end{aligned}
$$

where the Newton's constant $G_{N}$ is given by

$$
G_{N}=\frac{k \kappa_{5}^{2}}{16 \pi}
$$

Eqs. (23) are the same as equations in the standard cosmology, provided that the lower signs are taken and the terms linear in $a^{-4}$ are absorbed in $\rho$ and $p$. Therefore, the lower signs should be taken in all equations.

If the arbitrary constant $C$ is zero, then the low-energy evolution equations of $a(t)$ completely agree with equations in the standard cosmology. However, if $C$ is not zero, then new terms appear in the evolution equations. We shall now investigate how these terms can be interpreted. Eqs. (23) can be written as

$$
\begin{aligned}
H^{2} & =\frac{8 \pi G_{N}}{3} \rho_{e f f}, \\
\frac{\partial_{t}^{2} a}{a} & =-\frac{4 \pi G_{N}}{3}\left(\rho_{e f f}+3 p_{e f f}\right),
\end{aligned}
$$

where

$$
\begin{aligned}
& \rho_{e f f}=\rho-\frac{3 C k}{2 \kappa_{5}^{2}} \frac{1}{a^{4}}, \\
& p_{e f f}=p-\frac{1}{3} \frac{3 C k}{2 \kappa_{5}^{2}} \frac{1}{a^{4}} .
\end{aligned}
$$

Thus, the terms linear in $a^{-4}$ can be considered as a shift of radiation density. Since non-zero value of $C$ does not implies real existence of radiation but changes cosmological evolution equations, it can be understood as "dark radiation". Here, note that the constant $C$ can be both positive and negative, depending on the geometry in bulk. To be precise, the term proportional to $C$ can be understood as the 'electric' part of the 5-dimensional Weyl tensor in the general frame work developed in Ref. [9]. Moreover, it can be shown that if $C$ is positive then there appears a naked singularity in 5-dimension [17]. Thus, the constant $C$ should be zero or negative, which implies that energy density of the dark radiation should be zero or positive.

In summary, we have obtained new exact solutions of brane-world cosmology. These solutions include an arbitrary constant $C$. The constant $C$ is determined by the geometry outside the brane and affects the cosmological evolution in the brane-world. If $C$ is zero, then the standard cosmology governs the brane-world as a low-energy effective cosmological theory. However, if $C$ is not zero, then even in low-energy the brane-world cosmology gives predictions different from the standard one. The difference can be understood as "dark radiation", which is not real radiation but alters cosmological evolutions. Density of the dark radiation should be zero or positive.

Note added in proof: After this paper had been submitted, the author became aware of the works [18 21], in which similar solutions were obtained.

The author would like to thank Professor W. Israel for helpful discussions and continuing encouragement. The author is supported by the CITA National Fellowship and the NSERC operating research grant.

[1] M. B. Green, J. H. Schwartz, E. Witten, Superstring theory (Cambridge University Press, 1987).

[2] J. Polchinski, String Theory IE II (Cambridge University Press, 1998).

[3] E. Witten, Nucl. Phys. B443, 85 (1995).

[4] P. Hořava and E. Witten, Nucl. Phys. B460, 506 (1996); Nucl. Phys. B475, 94 (1996).

[5] I. Antoniadis and M. Quirós, Phys. Lett. B392, 61 (1997).

[6] E. Duras and C. Grojean, Nucl. Phys. B507, 553 (1997). 
[7] L. Randall and R. Sundrum, hep-th/9905221; hep-th/9906064.

[8] N. Kaloper, hep-th/9905210; T. Nihei, hep-ph/9905487; P. Binétruy, C. Deffayet and D. Langlois, hep-th/9905012: C. Csáki, M. Graesser, C. Kolda and J. Terning, hep-ph/9906513; J. M. Cline, C. Grojean and G. Servant, hep-ph/9906523; N. Kaloper, hep-th/9905210; E. E. Flanagan, S. H. H. Tye, I. Wasserman, hep-ph/9910498; H. B. Kim and H. D. Kim, hep-th/9909053.

[9] T. Shiromizu, K. Maeda and M. Sasaki, gr-qc/9910076.

[10] A. Chamblin, S. W. Hawking and H. S. Reall, hep-th/9909205.

[11] R. Emparan, G. T. Horowitz and R. C. Myers, hep-th/9911043.

[12] A. Chamblin and G. W. Gibbons, hep-th/9909130.

[13] J. Garriga and T. Tanaka, hep-th/9911055.

[14] S. S. Gubser, hep-th/9912001.

[15] W. Israel, Nuovo Cim. 44B, 1 (1966).

[16] A. Lukas, B. A. Ovrut, K. S. Stelle and D. Waldram, hep-th/9803235.

[17] S. Mukohyama, T. Shiromizu and K. Maeda, in preparation.

[18] P. Kraus, hep-th/9910149.

[19] P. Binétruy, C. Deffayet, U. Ellwanger and D. Langlois, hep-th/9910219.

[20] D. N. Vollick, hep-th/9911181.

[21] D. Ida, gr-qc/9912002. 